\title{
RECURSOS PSICOLÓGICOS E AJUSTAMENTO PESSOAL FRENTE À INCAPACIDADE FUNCIONAL NA VELHICE ${ }^{1}$
}

\author{
Dóris Firmino Rabelo* \\ Anita Liberalesso Neri"
}

\begin{abstract}
RESUMO. Diante do aumento da prevalência de condições crônicas na população idosa, crescente atenção é dada à identificação dos fatores psicológicos e sociais que podem afetar o bem-estar e a qualidade de vida de indivíduos mais velhos com incapacidades. Os recursos psicológicos e sociais de que o indivíduo dispõe são um caminho na determinação das implicações da incapacidade funcional na vida das pessoas afetadas. Esta revisão tem como objetivo apresentar algumas maneiras pelas quais os indivíduos enfrentam suas perdas em funções físicas e papéis sociais e a extensão em que os recursos psicológicos e sociais operam sobre os efeitos negativos das condições crônicas na qualidade de vida dos idosos. Entre as variáveis explanatórias apresentadas estão o suporte social, as crenças e estados emocionais positivos, a regulação afetiva, o mecanismo de comparação social, o senso de auto-eficácia percebida, o mecanismo de seleção-otimização-compensação e mecanismos de coping.
\end{abstract}

Palavras-chave: idosos, incapacidade funcional, ajustamento psicológico.

\section{PSYCHOLOGICAL RESOURCES AND PERSONAL ADJUSTMENT FOR FUNCTIONAL INCAPACITY IN AGING}

\begin{abstract}
Considering the increase of chronic conditions in the elderly people, more attention has been given to the identification of the psychological and social factors that can affect the well-being and the quality of life of individuals with incapacities. The psychological and social resources that the individual disposes are clues to determine the implications of the functional incapacity in the life of affected people. This review has the objective to present some ways which the individuals face their losses in physical functions and social roles, and in which extension the psychological and social resources operate over the negative effects of the chronic conditions in the elderly quality of life. Among the explainable variables presented there is the social support, the positive emotional states and beliefs, the affectionate regulation, the mechanism of social comparison, the sense of self-sufficiency, the selection-optimization-compensation mechanism and the coping mechanisms.
\end{abstract}

Key words: elderly, functional incapacity, psychological adjustment.

O envelhecimento populacional e o aumento da prevalência de doenças crônicas acarretaram $o$ crescimento das taxas de incapacidades físicas e/ou mentais entre os idosos brasileiros, fato que representa muitos desafios para as famílias e para a sociedade, além de ser um risco à boa qualidade de vida destes indivíduos mais velhos (Medina, Ramos, 2003; Sairassu \& Goldfeder, 1998). Segundo Karsch (2003), estima-se que no Brasil 10\% das pessoas acima de 65 anos precisam de ajuda em atividades básicas de autocuidado e que cerca de $40 \%$ necessitam de auxílio nas atividades instrumentais de vida diária, o que significa mais de 6 milhões de pessoas e famílias com necessidade de apoio e suporte.

A incapacidade funcional é a maior conseqüência das condições crônicas, além de afetar o status psicológico e o uso de serviços de cuidado de longa permanência. Para Lamb (1996), a incapacidade refere-se a problemas no funcionamento social e no desempenho de atividades normais da vida diária e de papéis socialmente definidos dentro de um ambiente particular sociocultural e físico. Segundo Jette (1996), compreender a incapacidade é incluir o exame das características da situação em que a pessoa funciona,

\footnotetext{
1 Apoio: FAPESP.

* Psicóloga. Mestranda em Gerontologia pela Universidade Estadual de Campinas-Unicamp.

\# Livre-docente. Professora Titular, vinculada ao Programa de Pós-graduação em Gerontologia, Faculdade de Educação da Universidade Estadual de Campinas-Unicamp.
} 
considerando não apenas as capacidades, mas também a relação da pessoa com aspectos relevantes da situação. Nesse sentido, considera-se como o indivíduo define a situação da incapacidade e reage a ela, como os outros definem a situação de incapacidade e colocam expectativas para os idosos e consideram-se também as características do próprio ambiente físico. O tópico da incapacidade funcional é de grande interesse não apenas porque esta é influenciada por condições médicas, patologia ou deficiência, mas também porque é influenciada por fatores psicológicos e sociais.

Como referido em estudo de Lima-Costa, Barreto e Giatti (2003) sobre as condições de saúde e capacidade funcional da população idosa brasileira, segundo a Pesquisa Nacional por Amostra de Domicílios (PNAD) de 1998, 69\% dos participantes relataram ter pelo menos uma doença crônica (sendo esta proporção maior entre as mulheres); por outro lado, a presença destas doenças, a prevalência de incapacidade funcional e a ocorrência de internações hospitalares aumentaram com a idade. Para Ormel, Kempen, Penninx, Brilman, Beekman e Sonderen (1997), indivíduos com condições médicas crônicas são mais propensos a experienciar sintomas depressivos e são menos capazes de controlar muitos aspectos de suas vidas. Além disso, à medida que a prevalência de muitas condições crônicas aumenta com a idade, os idosos vão adquirindo potencial risco de crescente distresse psicológico e de um funcionamento psicológico deficiente.

Os recursos psicológicos e sociais de que o indivíduo dispõe são um caminho na determinação das implicações da incapacidade funcional na vida das pessoas afetadas. Os fatores psicológicos refletem a percepção subjetiva do indivíduo e sua avaliação da situação, são importantes na adaptação à incapacidade, funcionam como recursos de enfrentamento, atenuando a adversidade de situações estressantes, e auxiliam no manejo do ambiente social e físico. Além dos recursos médicos, os fatores psicossociais têm grande potencial para determinar em que medida a vida estendida será vivida eficazmente ou com debilidade, dor e dependência (Bandura, 2004).

É importante a compreensão dos meios pelos quais os indivíduos enfrentam suas perdas em funções físicas e papéis sociais (Ormel \& cols., 1997). Entre aqueles afligidos pelas doenças e condições crônicas, alguns avançam para incapacidades severas e outros não. Tem-se pouco entendimento dos fatores protetores que moderam o impacto dessas condições entre os idosos. Diante do aumento da prevalência de condições médicas crônicas na população idosa, crescente atenção agora é dada diretamente à identificação dos fatores psicológicos e socioculturais que podem atuar de forma negativa no bem-estar e na qualidade de vida de indivíduos mais velhos com incapacidades.

Esta revisão tem como objetivo comentar estudos que tratam das maneiras como os indivíduos enfrentam suas perdas em funções físicas e papéis sociais e a extensão em que os recursos psicológicos e sociais operam sobre os efeitos negativos das condições crônicas na qualidade de vida dos idosos. Serão apresentadas algumas possíveis variáveis explanatórias de natureza psicológica e social que podem operar como mecanismos protetores ou mediadores no ajustamento pessoal de indivíduos mais velhos com incapacidades.

\section{QUALIDADE DE VIDA EM IDOSOS COM INCAPACIDADE FUNCIONAL}

Questões como o que é uma boa vida e como deveríamos viver para alcançar esta condição vêm sendo discutidas pela humanidade há centenas de anos. Definiçõos e indicativos de qualidade de vida podem variar desde o status socioeconômico, a satisfação de necessidades e a capacidade funcional até o sentido da vida, a satisfação com a vida, o bemestar e a felicidade. Diante dos vários modelos e definições de qualidade de vida na velhice, pode-se dizer que a experiência de uma doença que tem potencial para gerar déficits no funcionamento físico, sensorial e cognitivo afeta o desempenho cotidiano do indivíduo e a avaliação subjetiva que este faz de sua vida (Melzer \& Parahyba, 2004).

Para Freire (2003), as dimensões física, psicológica, social e espiritual devem ser consideradas e uma boa qualidade de vida implica em um indivíduo autônomo e independente, com boa saúde física, com senso de significado pessoal, desempenhando papéis sociais e permanecendo ativo. Paschoal (2004), em apresentação de parte de sua pesquisa de construção de um instrumento de qualidade de vida para idosos, considerou a diferença entre as expectativas do indivíduo e sua realização. A boa qualidade de vida envolve quanto o indivíduo realizou daquilo que considera importante, a distância entre o idealizado e o realizado e a satisfação com o que foi possível concretizar até o momento. As dimensões de qualidade de vida consideradas importantes para os idosos pesquisados foram a saúde (estado de saúde, doenças, deficiências dos sentidos, viver sem dor importante, etc.), a capacidade funcional (dependência em geral, AVDs, AIVDs, controle sobre a própria 
vida, etc.), a dimensão psicológica (alegria, tristeza, gostar de si mesmo, aproveitar a vida, etc.), a dimensão social (abandono da família, ser lembrado pelos filhos, os filhos estarem bem, etc.), a econômica (aposentadoria, ter casa própria, ter lugar para morar, etc.) e a ambiental (violência, poluição, etc.), hábitos e estilos de vida (tabagismo, elitismo, atividade física, etc.) e a espiritualidade.

A deficiência na competência comportamental tem impacto no bem-estar e na necessidade de ajuda informal e serviços de saúde dos idosos. Segundo Lawton (1991), a competência comportamental representa a avaliação socionormativa do funcionamento da pessoa quanto à saúde, ao uso do tempo e às dimensões sociais e cognitivas. Estas competências no dia-a-dia se refletem em autonomia e independência, levando-se sempre em conta os aspectos objetivos, subjetivos e contextuais. Elas manifestam-se na vivência e no comportamento em contextos cotidianos e abrangem aptidões físicas, cognitivo-emocionais e sociais em inter-relação com as possibilidades do ambiente. Segundo Baltes (1996), a dependência comportamental é a mais temida pelos idosos, pois os segrega de outros grupos etários e faz que eles sejam tidos como incompetentes de acordo com normas sociais, além de ser um desafio para o qual é preciso enfrentamento.

Em pesquisa buscando investigar os efeitos das doenças de longa permanência sobre a qualidade de vida de idosos britânicos, Ayis, Goodberman-Hill e Ebrahim (2003) encontraram que as limitações decorrentes dessas doenças estavam associadas com distresse psicológico, com vulnerabilidade percebida, com percepções mais negativas da própria saúde e falta de expectativa de uma vida longa. Segundo os autores, os domínios sociodemograficos, o ambiente físico e social, as condições da doença, o suporte social e os aspectos psicológicos (ex. solidão, senso de controle e de vulnerabilidade) são prováveis variáveis explanatórias dos efeitos limitantes da doença de longa permanência.

As doenças crônicas e as incapacidades conseqüentes podem afetar significativamente o bemestar dos idosos. Segundo Kahn e Juster (2002), a maior parte dos estudos que se preocuparam em acessar o bem-estar incluíram uma ou mais questões sobre a saúde. Para os autores, dado o aumento da morbidade e a comorbidades que freqüentemente aparecem com o avançar da idade, a redução na satisfação com a saúde fica evidente. Em estudo com homens de 50 a 72 anos hipertensos e com alto risco de doença cardiovascular, Agewall, Wikstrand, Dahlöf e Fagerberg (1996) encontraram que pacientes com qualquer doença cardiovascular sentem-se significativamente mais descontentes quando comparados com pacientes sem sinais ou sintomas de doenças cardiovasculares.

Clarke (2003), investigando idosos canadenses residentes na comunidade que haviam sofrido acidente vascular cerebral, um evento agudo que deixa seqüelas de longa permanência, encontrou que as incapacidades físicas e cognitivas estão sistematicamente associadas com menor senso de ajustamento psicológico após o AVC. Segundo o autor, a extensão em que a incapacidade restringe o senso pessoal de self poderia ser um caminho através do qual estas incapacidades afetam o ajustamento. Analisando saúde e bem-estar subjetivo entre idosos a partir dos dados do Berlim Aging Study, Smith, Borchelt, Maier e Jopp (2002) verificaram que doenças crônicas e deficiências funcionais limitam o bem-estar especialmente entre os idosos mais velhos (80 anos e mais). Segundo os autores, as cumulativas tensões crônicas de saúde limitam o potencial dos indivíduos mais velhos de experienciar o lado positivo da vida.

Segundo Xavier, Ferraz, Marc, Escostequy e Moriguchi (2003), para os idosos octogenários residentes em comunidade da cidade de Veranópolis, a falta de saúde é o principal indicador da ausência de bem-estar e de uma qualidade de vida negativa. Bryant, Corbett e Kutner (2001) investigaram o que constitui a saúde e contribui para isso na perspectiva dos idosos pesquisados, e daí emergiu um modelo de envelhecimento saudável. Para os idosos investigados, saúde significa fazer alguma coisa significativa, o que requer quatro componentes: algo que valha a pena fazer; equilíbrio entre habilidades e desafios; recursos sociais apropriados; e características atitudinais pessoais (ex. atitudes positivas X “pobre de mim”).

A maior incidência de eventos negativos na velhice - como doenças, perdas e acidentes - levanta a questão de como os idosos lidam de maneira bemsucedida com eles e conseguem manter uma boa qualidade de vida, ou pelo menos a melhor possível (Kahn \& Juster, 2002).

A seguir serão exploradas as maneiras como os indivíduos enfrentam suas perdas em funções físicas e papéis sociais e a extensão em que os recursos psicológicos e sociais operam sobre os efeitos negativos das condições crônicas na qualidade de vida dos idosos. Serão propostas algumas possíveis variáveis explanatórias de natureza psicológica e social que podem operar como mecanismos protetores ou mediadores em indivíduos mais velhos com incapacidades. 


\section{RECURSOS PSICOLÓGICOS E SOCIAIS ENVOLVIDOS NO AJUSTAMENTO PESSOAL FRENTE ÀS INCAPACIDADES NA VELHICE}

Vivenciar um evento negativo, como ficar incapacitado, que representa ameaça ao senso de controle pessoal e traz conseqüências numerosas e de longo prazo na vida do indivíduo, tende a acionar seus recursos pessoais e sociais. Segundo Fortes e Neri (2004), eventos inesperados relacionados à saúde, que ocorrem com mais freqüência na velhice, exigem da pessoa um grande esforço adaptativo e propõem uma demanda à personalidade individual, orientando-a ao enfrentamento dos desafios provenientes de um evento não desejado e ao ajustamento psicológico e social; por isso acionam intensamente os recursos emocionais e cognitivos da pessoa afetada, sendo o sucesso na adaptação das demandas exigidas pelo evento um importante indicador de bem-estar (Davies, 1996). Quanto mais desenvolvidos os mecanismos de ajustamento psicológico, maior a chance de adaptação, presumivelmente sem grande declínio na qualidade de vida do idoso. Para Silva e Varela (1999), adaptação significa maximizar as possibilidades individuais reorganizando a vida frente às limitações percebidas, ajustando-se às diversas situações individualmente ou com a ajuda de outros. É um processo contínuo de atualização das potencialidades pessoais e de aprender a viver com as limitações explorando e utilizando ao máximo seus recursos disponíveis.

A crise decorrente da ocorrência de uma incapacidade que demanda efetiva mudança traz dificuldades ao ajustamento pessoal, uma vez que acarreta interrupção na continuidade do estilo de vida, torna necessária a utilização de mais métodos de enfrentamento e de mais esforços para manter o senso de controle, faz com que mude a referência grupal e torna mais difícil identificar novos selves possíveis (Coleman, 1996). Para Davies (1996), as variáveis mais significativas envolvidas na adaptação são o suporte social, o coping e o controle percebido.

De acordo com Antonucci (2001), o suporte social ajuda os indivíduos a enfrentar e se recuperar das demandas da vida. Segundo a autora, pessoas com amplas redes sociais têm mais ajuda nos tempos de doenças e as pessoas que percebem mais suporte enfrentam melhor as enfermidades, o estresse e outras experiências difíceis da vida. Para Handen (1991), o suporte social aumenta a auto-estima e o sentimento de domínio sobre o próprio ambiente, tem a função de coping ao amenizar o impacto das doenças e provavelmente modera o efeito das crises inesperadas. Segundo Antonucci (1994), o suporte social estimula o senso de controle e auto-eficácia. Os membros da rede social são capazes de promover no indivíduo a crença de competência e de capacidade de controlar seu ambiente e ser bem-sucedido. As mulheres têm redes sociais maiores e mais multifacetadas, percebem melhor disponibilidade de suporte, dão mais suporte e têm melhores habilidades interpessoais do que os homens. Na velhice, essas habilidades tornam-se significativamente mais úteis na expansão e no fortalecimento das redes de suporte social. Além disso, as mulheres fazem melhor uso de instituições socialmente organizadas (Antonucci, 2001; Haden, 1991).

Os eventos da vida podem ser estressantes porque exigem ajustamento da parte do indivíduo e também porque restringem fontes importantes de suporte, o que tem grande impacto sobre a saúde e o bem-estar. Em estudo objetivando identificar os fatores da qualidade de vida associados com os padrões de incapacidade na velhice, Lamb (1996) verificou que os indivíduos mais incapacitados funcionalmente e com perfis mais depressivos (tendência maior para mulheres) apresentaram mais saúde percebida negativamente, escores de humor mais baixos e menor satisfação com a freqüência de contato e de visitas de familiares e amigos. Aqueles muito deprimidos tinham menor suporte instrumental em termos de parentes disponíveis.

$\mathrm{Na}$ tentativa de identificar os determinantes mais importantes de bem-estar entre idosos poloneses após a ocorrência do primeiro AVC isquêmico, Jaracz e Kozubski (2003) encontraram que o mais forte é a extensão do suporte emocional, seguido da presença de depressão e do status funcional. Segundo Agewall, Wikstrand, Dahlöf e Fagerberg (1996), o aumento da mortalidade em pacientes hipertensos com alto risco de doença cardiovascular esteve associado com uma rede social pobre. De acordo com Clarke (2003), para os sobreviventes de AVC participantes de sua pesquisa o suporte social apareceu como importante para o senso positivo de bem-estar e para o domínio sobre o ambiente, em parte porque ajuda nas estratégias de adaptação das incapacidades. Aqueles com uma grande rede de suporte social percebiam grandes oportunidades de crescimento pessoal e aqueles que estavam satisfeitos com seu suporte tiveram escores mais altos nas relações positivas com outros e na auto-aceitação.

Recursos psicológicos como o otimismo, o controle pessoal e o senso de significado também são importantes como reservas que permitem às pessoas enfrentar mais efetivamente os eventos críticos da vida. Segundo Taylor, Kemeny, Reed, Bower e Grunewald (2000), ilusões positivas que 
representam crenças positivas moderadas de distorções sobre a realidade, como o otimismo irreal e a percepção exagerada de controle pessoal, têm efeitos psicológicos protetores. Segundo os autores existem algumas razões pelas quais as ilusões positivas sobre a saúde podem inclusive afetar o curso da doença física. Em primeiro lugar as crenças positivas têm impacto nos estados emocionais, que por sua vez podem causar mudanças fisiológicas e neuroendócrinas. Elas também podem promover comportamentos saudáveis, uma vez que pessoas dotadas de senso de autovalor acreditam no seu poder de controle e são otimistas quanto ao seu futuro; são também mais propensas a praticar hábitos de saúde mais conscientes e a fazer uso dos serviços mais apropriadamente. Para Ramos (2003), embora grande parte dos idosos tenha pelo menos uma doença crônica, se estes mantiverem suas enfermidades controladas são capazes de levar uma vida normal mesmo diante de incapacidades. A terceira razão que conecta as crenças positivas ao curso da doença é baseada no fato de que os estados emocionais positivos estão associados com bons relacionamentos sociais. Finalmente, o otimismo, o senso de controle pessoal e a autoestima estão ligados aos esforços ativos de coping, e as ilusões positivas ajudam os indivíduos a encontrar significado na experiência. Para Salovey, Rothman, Detweiler e Steward (2000), as experiências emocionais positivas aumentam a disponibilidade de recursos psicológicos, uma vez que pessoas com estados emocionais positivos acreditam ser menos vulneráveis, enfrentam mais efetivamente os eventos estressantes e apresentam maior disponibilidade de contatos interpessoais e mais soluções criativas.

Labouvie-Vief e Medler (2002) acreditam que a regulação afetiva envolve duas estratégias independentes: a otimização afetiva e a complexidade afetiva. Os idosos têm grande capacidade de resiliência e, frente a perdas, tendem a organizar seu ambiente de maneira a maximizar afetos positivos e a amenizar os negativos. Essa habilidade auto-reguladora é chamada de otimização afetiva. Um critério adicional é a complexidade afetiva, caracterizada pela amplificação do afeto na busca por diferenciação e objetividade. Refere-se aos mecanismos de regulação emocional que envolvem a habilidade de coordenar afetos positivos e negativos em estruturas flexíveis e diferenciadas. No desenvolvimento humano, à medida que o sistema cognitivo amadurece, os indivíduos são mais capazes de coordenar sentimentos positivos e negativos através de processos de inibição/desinibição, avaliação e análise, e como resultado são desenvolvidas estruturas cognitivoafetivas complexas.

Os mesmos autores sugerem que a otimização afetiva e a complexidade afetiva cooperam para formar uma auto-regulação efetiva e que as estruturas cognitivo-afetivas podem declinar na complexidade da meia-idade em diante. Sua pesquisa mostrou que a dimensão da otimização afetiva está relacionada principalmente com a família e com a qualidade dos relacionamentos, e a dimensão da complexidade afetiva está relacionada com a complexidade do ambiente, o status econômico e a educação. Além disso, o afeto positivo e sua maximização têm diferentes significâncias no contexto de alta ou baixa complexidade afetiva.

A incapacidade oferece às pessoas muitas oportunidades de tirar conclusões sobre como sua situação se compara com a de outras pessoas (Tennen \& Afleck, 1997). A comparação com outros desempenha um papel importante na avaliação e construção da realidade e no enfrentamento de eventos negativos. Em situações que produzem um decréscimo no bem-estar, os indivíduos irão freqüentemente se comparar com outros que eles acreditam serem piores, em um esforço de melhorar seu bem-estar, particularmente quando não têm em vista oportunidades de ações instrumentais (Buunk, Gibbons \& Reis-Bergan, 1997). Esse mecanismo de enfrentamento é denominado por Wills (1997) de comparação descendente (downward comparison). A ciência, por parte da pessoa, de que existem indivíduos com problemas piores ou atributos inferiores e que há situações potencialmente piores, faz emergir a percepção de que seu status é relativamente mais favorável, e isso produz um aumento no bem-estar subjetivo. A comparação descendente poderá atuar reduzindo o afeto negativo atual e permitir que a pessoa sinta-se melhor no momento. Para Kulik e Mahler (1997), a afiliação a outros grupos que tenham passado pelo mesmo problema pode ajudar no enfrentamento da situação. Segundo os autores, argumenta-se que em geral a decisão de se juntar a esses grupos envolve o processo de comparação social. O contato com outros que estão enfrentando situações similares reduz a ansiedade e os 
sentimentos de singularidade ou de desviante, além de ser fonte de suporte social. A proliferação de tais grupos nos últimos anos tem demonstrado por si só a sua importância no enfrentamento de situações estressantes.

Entre os atributos psicológicos importantes para o gerenciamento de condições crônicas está a crença de auto-eficácia percebida. O conceito de auto-eficácia, introduzido por Bandura no contexto da teoria social cognitiva, diz respeito às crenças que as pessoas têm em sua própria capacidade de organizar e executar os cursos de ação requeridos para alcançar determinados resultados. A autocrença das pessoas em suas capacidades permite que elas exerçam controle sobre os eventos que afetam suas vidas; e como essas crenças se traduzem em realização e motivação, elas têm forte impacto no pensamento, no afeto, na motivação e na ação (Bandura, 1993).

A auto-eficácia percebida influencia o que as pessoas escolhem fazer, sua motivação, sua perseverança frente a dificuldades, sua vulnerabilidade ao estresse e à depressão. Influencia-as na situação de presença de doenças crônicas inclusive no nível de benefício que os indivíduos recebem das intervenções terapêuticas. Altos níveis de auto-eficácia para o enfrentamento das conseqüências das doenças crônicas estão associados com a melhora nos sintomas, melhor bem-estar físico e emocional e o aumento das atividades sociais. Assim, a auto-eficácia percebida é uma pré-condição essencial para o manejo apropriado da doença crônica (Holman \& Lorig, 1992). Baixas expectativas de auto-eficácia percebida podem predizer grande incapacidade, e para as pessoas idosas centram-se principalmente na reavaliação e na avaliação incorreta de suas capacidades. De fato, muitas capacidades físicas podem declinar na velhice, especialmente diante de doenças, requerendo reavaliações da eficácia pessoal. A avaliação da própria capacidade é altamente vantajosa e não raro essencial para o funcionamento efetivo. Aqueles que julgam incorretamente o que são capazes de fazer podem engajar-se em desempenhos que produzem conseqüências pouco satisfatórias (Bandura, 1997).

Segundo Baltes (1996), quando a velhice tornase cheia de restrições tanto na capacidade quanto nas oportunidades ambientais, os idosos invariavelmente lançam mão da seleção e compensação. Com o objetivo de manter e otimizar a funcionalidade, a dependência em alguns domínios tem que ser ajustada. A seleção, a otimização e a compensação (SOC) são três processos adaptativos. A seleção é invocada em resposta a perdas na funcionalidade ou capacidade, e inclui evitar, reduzir ou restringir o número de atividades, bem como reorganizar objetivos pessoais com vistas a focalizar aquelas áreas que são mais importantes ou salientes na vida cotidiana do indivíduo. Otimizar as atividades abarca os esforços que as pessoas fazem para ampliar ou enriquecer suas reservas com vista a permanecer funcionando, como, por exemplo, praticar, treinar e exercitar atividades para superar o declínio comportamental. Os processos de compensação são respostas às perdas na capacidade e incluem processos psicológicos ou esforços comportamentais para melhorar a funcionalidade. A manutenção da competência dependeria então da seleção dos domínios em que o indivíduo retém melhor nível de funcionamento, otimizando-os mediante estratégias de treino e garantindo assim a compensação das perdas.

Segundo Lang, Rieckmann e Baltes (2002), o uso de estratégias de seleção, otimização e compensação está associado com uma melhor funcionalidade física e mental e também requer o uso da capacidade individual nos domínios do funcionamento sensório-motor, cognitivo, social e da personalidade. Assim, quanto mais rico em recursos sensório-motores, cognitivos, sociais e de personalidade for o indivíduo que envelhece, melhor é o engajamento no uso de estratégias de seleção, otimização e compensação. No estudo realizado pelos autores com base no Berlin Aging Study, foi proposto que idosos ricos nestes recursos exibem menos diferenças negativas associadas ao envelhecimento e funcionam melhor no cotidiano do que aqueles que são pobres em recursos. Os resultados sugeriram que o uso de estratégias de seleção, otimização e compensação no cotidiano é maior entre os idosos ricos em recursos, quando comparados com aqueles pobres em recursos. Os idosos ricos em recursos sensório-motores, cognitivos, sociais e de personalidade investiam mais tempo com seus familiares, reduziam a diversidade de atividades dentro do domínio mais saliente de lazer, dormiam com mais freqüência e aumentaram a variabilidade de investimentos de tempo através de atividades. Em estudo que buscou investigar os esforços comportamentais de idosos com osteoartrite e/ou osteoporose usados na adaptação de suas incapacidades, Gignac, Cott e 
Badley (2000) verificaram que os idosos usam uma ampla variedade de formas de adaptação, que incluem a compensação de perdas, a otimização de performances, a restrição ou limitação de atividades, e ganham ajuda de outros.

Segundo Rothermund e Brandtstädter (2003), com o avançar da idade estes esforços compensatórios mais cedo ou mais tarde chegam ao seu limite. Acima dos 70 anos o decréscimo nesses esforços compensatórios está associado à diminuição da disponibilidade e da eficiência dos recursos de ação. Para os autores, a resiliência do self na velhice não pode ser explicada simplesmente em termos de esforços ativos de compensação. Os processos de enfrentamento assimilativo e acomodativo dão uma explicação mais compreensiva, que considera o efeito recíproco entre as intervenções ativas e o ajustamento de metas pessoais e padrões para mudanças nos recursos de ação. O enfrentamento assimilativo consiste na tentativa de evitar ou diminuir as perdas mediante atividades instrumentais, autocorretivas e compensatórias. Neste modo de enfrentamento, o indivíduo tenta transformar a situação avaliada negativamente de maneira a conformá-la com as aspirações e metas pessoais. Um modo diferente de neutralizar a discrepância entre o estado atual e o idealizado consiste em adaptar as metas e os objetivos aos dados constrangimentos. Esse processo acomodativo envolve o desengajamento e a diminuição dos padrões de performance pessoais e aspirações, isto é, os indivíduos acomodam seus objetivos e adotam padrões de performance mais suaves ou indulgentes. Os processos acomodativos são dominantes quando as repetidas tentativas ativas de mudança da situação são mal-sucedidas e são importantes para amenizar tensões emocionais que emergem diante da experiência de incapacidades irreversíveis. Os idosos participantes da pesquisa dos autores sobre a questão dos dois processos de coping, mostraram que a estabilidade na auto-avaliação depende do ajustamento de padrões pessoais, e que a aceitação de padrões mais indulgentes de comparação amenizaram os efeitos negativos dos déficits físicos na satisfação com a performance pessoal.

Como destacou Coleman (1996), a crise decorrente da ocorrência de uma incapacidade que exige mudança efetiva traz dificuldades de ajustamento, porque também torna mais difícil identificar novos selves possíveis. De acordo com Atcheley (1999), o desenvolvimento da incapacidade pode ter efeitos negativos nas várias dimensões do self, uma vez que altera a estrutura social do indivíduo no sentido de introduzir constrangimentos físicos e no estilo de vida, influencia negativamente a percepção individual de agência, reduz a concepção de uma pessoa resiliente emocionalmente e traz abandono de algumas metas pessoais. Para o autor (1991), inclui-se nos impactos negativos a interrupção da continuidade do self, mais necessidade de defesas e enfrentamento de perdas para o self, reduzidas capacidades de usar defesas como a interação seletiva, dificuldade de identificar selves possíveis, despersonalização do ambiente social, mudança nas referências grupais e menores habilidades em usar feedback na reconstituição do self. Os indivíduos incapacitados usualmente têm que redefinir o significado de competência e autoconfiança; no entanto, existem muitos indivíduos mais velhos que conseguem enfrentar positivamente a fragilidade. Encontrou-se em estudo feito por Atcheley (1999) que a maioria dos idosos pesquisados que desenvolveram incapacidades mostraram continuidade no senso de agência, na resiliência emocional e nas metas pessoais. Segundo o autor, estes resultados sugerem que o desejo interno por continuidade excede as pressões externas e situacionais por mudanças, pelo menos em relação a estes elementos do self e, na ausência de graves limitações e incapacidades, os idosos conseguem a continuidade nos vários domínios do self.

Segundo Staudinger, Marsiske e Baltes (1995), diante de perdas o self apresenta resiliência ou capacidade de reserva, isto é, o potencial de manutenção e recuperação dos níveis de adaptação normal e a presença de recursos latentes que podem ser ativados frente aos desafios e exigências. É proposto que os idosos, mesmo sob condições de limitações e incapacidades funcionais, mantêm um senso positivo de bem-estar (Smith, Borchelt, Maier \& Jopp 2002). Segundo Neri (2001), perdas na funcionalidade não são necessariamente um impedimento para a continuidade do funcionamento cognitivo e emocional, e como qualquer ser humano, o idoso consegue ativar mecanismos compensatórios para lidar com essas perdas. Para Clarke (2003), os processos psicológicos de adaptação e recursos sociais como programas de reabilitação e cuidados em domicílio são importantes na redução dos efeitos adversos das 
incapacidades funcionais sobre o bem-estar na idade avançada.

A capacidade dos indivíduos de se ajustar à diversidade e à dificuldade das experiências de vida, mantendo níveis positivos de bem-estar e qualidade de vida, apresenta um paradoxo. Segundo (Albrecht \& Devlieger, 1999), o paradoxo da incapacidade leva à questão: "Por que muitas pessoas com incapacidades sérias e persistentes relatam que experimentam boa ou até mesmo excelente qualidade de vida quando a maioria dos observadores externos destes indivíduos acredita que estes têm uma existência indesejável?" $\mathrm{Na}$ tentativa de explicar esta questão, os autores encontraram nos resultados de sua pesquisa a confirmação deste paradoxo e constataram também que, para os indivíduos com incapacidades entrevistados, a boa qualidade de vida depende do balanço entre corpo e mente e da manutenção de relações harmoniosas dentro dos contextos pessoais e sociais. Algumas pessoas incapacitadas são capazes de produzir e manter esse equilíbrio e conseqüentemente experimentar alta qualidade de vida, enquanto outros não são capazes de lidar com suas condições de saúde, têm limitados recursos, pouco conhecimento e restrições ambientais.

Eventos negativos como a incapacidade funcional decorrente de condições crônicas levantam a questão de como estes eventos afetam a qualidade de vida dos indivíduos e de como esses indivíduos lidam com eles de maneira bem-sucedida. À medida que as pessoas envelhecem, a habilidade de se recuperar de eventos negativos da vida adquire importância adicional (Kahn \& Juster, 2002). Considerando que o envelhecimento e o desenvolvimento pessoal são processos complexos dependentes do contexto sócio-histórico e cultural e da interação de fatores biológicos e subjetivos, chamamos a atenção para a importância de pesquisas mais aprofundadas sobre o que é ser velho no Brasil, com sua realidade sociocultural multifacetada, e como estes idosos estão se comportando frente às suas incapacidades e doenças. $\mathrm{O}$ envelhecimento satisfatório é dependente do equilíbrio entre as limitações e as potencialidades do indivíduo, e aqui foram propostos alguns recursos pessoais e sociais de que os indivíduos lançam mão frente a um evento indesejado, na tentativa de alcançar este equilíbrio. Diante do acelerado envelhecimento da população brasileira e de suas conseqüências, muitas possibilidades de estudo e prática se abrem para o psicólogo frente a este campo ainda muito inexplorado num país tão diverso quanto o nosso, e com muitas demandas clínicas, sociais e educacionais emergentes.

\section{CONSIDERAÇÕES FINAIS}

O fato de que ser idoso e incapacitado resulta em maior desvantagem social já está documentado; mas também é sabido que grande parte dos idosos são de alguma maneira capazes de neutralizar os efeitos destas desvantagens e manter satisfatória sua qualidade de vida, suas autoconcepções e seu ajustamento pessoal (Atcheley, 1999). Para tanto, é importante a disponibilidade de recursos psicológicos e sociais, entre eles, o suporte social, as crenças e estados emocionais positivos, a regulação afetiva, o mecanismo de comparação social, o senso de autoeficácia percebida, o mecanismo de seleçãootimização-compensação e mecanismos de coping.

$\mathrm{O}$ reconhecimento da longa permanência das consequiências psicossociais das condições incapacitantes é crucial no estabelecimento de programas de cuidado. Com o crescente número de idosos vivendo com doenças crônicas, problemas de saúde e decréscimo na capacidade, o que importa para os profissionais engajados nestas questões é ajudar esses idosos a viver uma vida tão boa quanto possível. É relevante ter em mente que quanto mais integrados psicológica e socialmente estiverem os idosos, menos ônus eles trarão para suas famílias e cuidadores e para os serviços de saúde e melhor será sua qualidade de vida.

\section{REFERÊNCIAS}

Agewall, S., Wikstrand, J., Dahlöf, C. \& Fagerberg, B. (1996). Negative feelings (discontent) predict progress of Intima-media thickness of the commom carotid artery in treated hypertensive men at high cardiovascular risk. American Journal of Hypertension, 9(6), 545-550.

Albrecht, G. L. \& Devilieger, P. J. (1999). The disability paradox: high quality of life against all odds. Social Science \& Medicine, 48, 977-988.

Antonucci, T. C. (1994). A life-span view of women's social relations. Em B. F. Turner \& L. E. Troll (Eds.). Women growing older: psychological perspectives, (pp. 239-269). Sage Publications.

Antonucci, T. C. (2001). Social relations: an examination of social networks. Em J. E. Birren \& K. W. Schaie (Eds.). Handbook of the Psychology of Aging, (pp. 427-453). San Diego: Academic Press.

Ayis, S., Goodberman-Hill, R. \& Ebrahim, S. (2003). Longstanding and limiting long-standing illness in older people: associations with chronic diseases, psychosocial and environmental factors. Age and Ageing, 32(3), 265-272.

Atcheley, R. C. (1991). The influence of aging or frailty on perceptions and expressions of the self: theoretical and methodological issues. Em J. E. Birren (Ed.). The Concept and Measurement of Quality of Life in the Frail Elderly, (pp. 207225). San Diego: Academic Press. 
Atcheley, R. C. (1999). Continuity theory, self, and social structure. Em C. D. Ryff \& V. C. Marshall (Eds.). The self and society in aging process, (pp. 122-149). New York: Springer.

Baltes, M. M. (1996). The many faces of dependency in old age. Cambridge University Press.

Bandura, A. (1993). Perceived self-efficacy in cognitive development and functioning. Educational Psychologist, 28, 117-148.

Bandura, A. (1997). Self-Efficacy: the exercise of control. New York: W. H. Freeman and Company.

Bandura, A. (2004). Health promotion by social cognitive means. Health Education \& Behavior, 31(2), 143-164.

Bryant, L. L., Corbett, K. K., \& Kutner, J. S. (2001). In their own words: a model of heathy aging. Social Science and Medicine, 53, 927-941.

Buunk, B. P., Gibbons, F. X. \& Reis-Bergan, M. (1997). Social comparision in health and illness: a historical overview. Em B. P. Buunk \& F. X. Gibbons. Health, Coping, and Well-Being: Perspectives from social comparision theory, (pp. 1-23). Mahwah, Lawrence Erlbaum Associates.

Clarke, P. (2003). Towards a greater understanding of the experience of stroke: integrating quantitative and qualitative methods. Journal of Aging studies, 17, 171-187.

Coleman, P. G. (1996). Identity management in later life. Em R. T. Woods (Ed.). Handbook of the Clinical Psychology of Ageing, (pp. 93-114). Chichester: John Wiley \& Sons.

Davies, A. D. M. (1996). Life events, health, adaptation and social support in the clinical Psychology of late life. Em R. T. Woods (Ed.). Handbook of the Clinical Psychology of Ageing, (pp. 115-140). Chichester: John Wiley \& Sons.

Fortes, A. C. G. \& Neri, A. L. (2004). Eventos de vida e envelhecimento humano. Em A. L. Neri, M. S. Yassuda \& M. Cachioni (Orgs.). Velhice bem-sucedida: aspectos afetivos $e$ cognitivos (pp. 51-70). Campinas, SP: Papirus.

Freire, S. A. (2003). Educação permanente e qualidade de vida na velhice - Meta e desafio na atualidade. Em $3^{\circ}$. Congresso Paulista de Geriatria e Gerontologia. Santos: SBGG- São Paulo (CD-ROM). Santos.

Gignac, N. A. M., Cott, C. \& Badley, E. M. (2000). Adaptation to chronic illness and disability and its relationship to perceptions of independence and dependence. Journal of Gerontology: Psychological Sciences, 55B (6), 362-372.

Handen, B. L. (1991). The influence of social support factors on the well-being of the elderly. Em P. A. Wisocki. Handbook of clinical behavior therapy with the elderly client (pp. 121139). New york: Plenum Press.

Holman, H. \& Lorig, K. (1992). Perceived self-efficacy in selfmanagement of chronic disease. Em R. Schwarzer. SelfEfficacy: thought control and action, (pp. 305-323). Taylor \& Francis.

Jaracz, K. \& Kozubski, W. (2003). Quality of life in stroke patients. Acta Neurologica Scandinavica, 107, 324-329.

Jette, A. M. (1996). Disability trends and transitions. Em R. H. Binstock \& L. K. George (Eds.). Handbook of Aging and the Social Science, (pp. 94-116). San Diego: Academic Press.

Kanh, R. L, \& Juster, F. T. (2002). Well-being: concepts and measures. Journal of Social Issues, 58(4), 618-627.
Karsch, U. M. (2003). Idosos dependentes: famílias e cuidadores. Cadernos de Saúde Pública, 19(3), 861-866.

Kulik, J. A. \& Mahler, H. I. M. (1997). Social comparision, affiliation, and coping with acute medical threats. Em B. P. Buunk \& F. X. Gibbons (Eds.). Health, Coping, and WellBeing: Perspectives from social comparision theory, (pp. 227-262). Mahwah, Lawrence Erlbaum Associates.

Labouvie-Vief, G. \& Medler, M. (2002). Affect optimization and affect complexity: modes and styles of regulation in adulthood. Psychology and Aging, 17(4), 571-588.

Lamb, V. L. (1996). A cross-national study of quality of life factors associated with patterns of elderly disablement. Social Science and Medicine, 42(3), 363-377.

Lang, F. R., Rieckmann, N. \& Baltes, M. M. (2002). Adapting to aging losses: do resources facilitate strategies os selection, compensation, and otimization in everyday functioning? Journal of Gerontology: Psychological Sciences, 57B(6), 501-509.

Lawton, M. P. (1991). A multidimensional view of quality of life in frail elders. Em J. E. Birren (Ed.). The Concept and Measurement of Quality of Life in the Frail Elderly (pp. 3-27). San Diego: Academic Press.

Lima-Costa, M. F., Barreto, S. M. \& Giatti, L. (2003). Condições de saúde, capacidade funcional, uso de serviços de saúde e gastos com medicamentos da população idosa brasileira: um estudo descritivo baseado na Pesquisa Nacional por Amostra de Domicílios. Cadernos de Saúde Pública, 19(3), 735-743.

Medina, M. C. G., Sairassu, M. M. \& Goldfeder, M. C. (1998). Das incapacidades e do Acidente Vascular Cerebral. Em U. M. Karsch (Org.). Envelhecimento com dependência: revelando cuidadores, (pp. 199-214). São Paulo: Educ.

Melzer, D. \& Parahyba, M. I. (2004). Sócio-demografic correlates of mobility disability in older brazilians: results of the first national survey. Age and Ageing.

Neri, A. L. (2001). Velhice e qualidade de vida na mulher. Em A. L. Néri (Org.). Desenvolvimento e envelhecimento: perspectivas biológicas, psicológicas e sociológicas, (pp. 161200). Campinas, SP: Papirus.

Ormel, J., Kempen, G. I., Penninx, B. W. J. H., Brilman, E. I., Beekman, A. T. F. \& Sonderen, E. (1997). Chronic medical conditions and mental health in older people: disability and psychosocial resources mediate specific mental health effects. Psychological Medicine, 27, 1065-1077.

Paschoal, S. M. C. (2004). Avaliação de qualidade de vida. Mesa redonda apresentada no XIV Congresso Nacional de Geriatria e Gerontologia, Salvador, Bahia.

Ramos, L. R. (2003). Fatores determinantes do envelhecimento saudável em idosos residentes em centro urbano: Projeto Epidoso, São Paulo. Cadernos de Saúde Pública, 19(3), 793798.

Rothermund, K. \& Bradtstädter, J. (2003). Coping with deficits and losses in later life: from compensatory action to accommodation. Psychology and Aging, 18(4), 896-905.

Salovey, P., Rothman, A. J., Detweiler, J. B. \& Steward, W. T. (2000). Emotional states and physical health. American Psychologist, 55(1), 110-121. 
Silva, M. J. \& Varela, Z. M. (1999). O conceito de adaptação na terceira idade: uma aproximação teórica. Arquivos de Geriatria e Gerontologia, 3(1), p. 25-29.

Smith, J., Borchelt, M., Maier, H. \& Jopp, D. (2002). Health and well-being in the young and oldest old. Journal of Social Issues, 58(4), 715-732.

Staudinger, U. M., Marsiske, M. \& Baltes, P. B. (1995). Resiliência e níveis de capacidade de reserva na velhice: perspectivas da teoria de curso de vida. Em A. L. Neri (Org.). Psicologia do Envelhecimento: temas relacionados na perspectiva de curso de vida, (pp.195-228). Campinas: Papirus.

Taylor, S. E., Kemeny, M. E., Reed, G, M., Bower, J. E. \& Grunewald, T. L. (2000). Psychological resources, positive ilusions, and health. American Psychologist, 55(1), 99-109.

Tennen, H. \& Affleck, G. (1997). Social comparision as a coping process: a critical review and aplication to chronic pain disorders. Em B. P. Buunk \& F. X. Gibbons. Health, Coping, and Well-Being: Perspectives from social comparision theory, (pp. 263-298). Mahwah, Lawrence Erlbaum Associates.

Wills, T. A. (1997). Modes and families of coping: an analysis of social comparision in the structure of other cognitive and behavioral mechanisms. Em B. P. Buunk \& F. Gibbons (Eds.). Health, Coping, and Well-Being: Perspectives from social comparision theory, (pp. 167-194). Mahwah: Lawrence Erlbaum Associates.

Xavier, F. M. F., Ferraz, M. P. T., Marc, N., Escostequy, N. U. \& Moriguchi, E. H. (2003). Elderly people's definition of quality of life. Revista Brasileira de Psiquiatria, 25(1), 310-317.

Recebido em 27/05/2005 Aceito em 15/09/2005
Endereço para correspondência:

Dóris Firmino Rabelo, Rua Professora Guaraciaba, 246. Bairro Tabajaras. CEP 38400-266 Uberlândia-MG. E-mail: dorisedmar@ centershop.com.br 